With compliments of the Author 


\title{
Organoselenosilane-Mediated Selective Mild Access to Selenolesters, Selenoanhydrides and Diacyl Diselenides
}

\author{
Antonella Capperucci,* Alessandro Degl'Innocenti,* Caterina Tiberi \\ Department of Chemistry 'Ugo Schiff', University of Florence, Via della Lastruccia 3-13, 50019 Sesto Fiorentino (Firenze), Italy \\ Fax +39(055)4573585; E-mail: alessandro.deglinnocenti@ unifi.it \\ Received 20 June 2011
}

Dedicated to Professor Alfredo Ricci with admiration, respect, and best wishes on the occasion of his retirement

\begin{abstract}
Reaction of acyl chlorides with phenylselenotrimethylsilane promoted by TBAF afforded a mild general access to selenolesters in good yields. When acyl chlorides were reacted with bis(trimethylsilyl)selenide (HMDSS) in 2:1 or 1:1 ratio a selective entry to selenoanhydrides or diacyl diselenides respectively was obtained.
\end{abstract}

Key words: selenosilanes, selenolesters, diacyl selenides, diacyl diselenides, fluoride ion

The chemistry of organoselenium compounds has recently faced a strong development, due to the fact that several compounds of this class have been demonstrated to be versatile reagents in organic synthesis ${ }^{1}$ and, more recently, in asymmetric catalysis. ${ }^{2}$ They also have been efficiently used in the synthesis of molecules with biological activity, ${ }^{3}$ steroids and sex hormones. ${ }^{4}$

In this context selenolesters have been shown as useful intermediates in synthetic organic chemistry. These molecules have in fact been used as precursors of acyl radicals ${ }^{5}$ and anions, ${ }^{6}$ reagents for the transfer of acyl moieties ${ }^{7}$ in rather mild conditions, in the synthesis of ketones ${ }^{8}$ and asymmetric aldol reactions. ${ }^{9}$ Various synthetic methods for selenolesters have been reported and usually are based on the reactions of acyl chlorides or acid anhydrides with selenols, ${ }^{10}$ diselenides ${ }^{11}$ or their metal selenoates, ${ }^{12}$ as well as aryl tributylstannyl selenides ${ }^{13 \mathrm{a}-\mathrm{d}}$ and phenylselenotris(trimethylsilyl)silane ${ }^{13 e}$ have been used. Alkenyl and alkynyl selenides behave as efficient precursors, ${ }^{14}$ while selenocyanates and tributylphosphine have been used to produce selenolesters from carboxylic acids. ${ }^{15}$

In addition, several papers have recently appeared on their synthesis mediated by indium metal, ${ }^{16}$ or in ionic liquids. ${ }^{17}$ Some other methods are also present in the literature. $^{18}$

We have been interested for a long time in the generation of synthetic equivalents of nucleophilic species through the functionalization of the $\mathrm{C}-\mathrm{Si}$ bond. ${ }^{19}$

In this context we recently reported the reactivity of silylated dithiolanes toward different electrophiles, ${ }^{20}$ thus showing that such molecules can behave as synthetic

SYNLETT 2011, No. 15, pp 2248-2252

Advanced online publication: 24.08.2011

DOI: 10.1055/s-0030-1261195; Art ID: S05511ST

(C) Georg Thieme Verlag Stuttgart · New York equivalents of a dithiolane carbanion, a species that cannot be directly generated upon metalation.

In addition, the functionalization of the $\mathrm{S}-\mathrm{Si}$ bond led us to access thiocarbonyl compounds, ${ }^{21}$ or more recently to transfer a thiol moiety onto ring-strained heterocycles such as epoxides. ${ }^{22}$ In recent years also the reactivity of the $\mathrm{Se}-\mathrm{Si}$ bond was explored in the possible generation of selenocarbonyl compounds, ${ }^{23}$ seleno heterocycles and diselenides. ${ }^{24}$

In this context, we thought that selenosilanes could behave efficiently as synthetic equivalents of unstable selenols, capable of being easily handled and to release, upon treatment with appropriate catalysts, nucleophilic species, able to transfer a seleno group onto different electrophiles. With this concept in mind we first wanted to test the ability of selenosilanes in transferring an organo-selenyl moiety, and we reacted benzoyl chloride (1a) with $\mathrm{PhSeSiMe}$ (2) under the influence of fluoride ion as a test reaction (Scheme 1). The reaction proved quite efficient affording as the only product the selenolester 3a in almost quantitative yield (95\%; Scheme 1).<smiles>O=C(Cl)c1ccccc1</smiles>
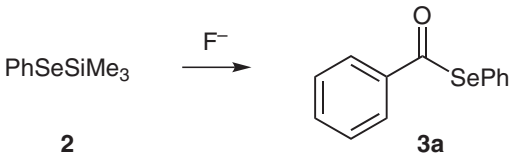

Scheme 1

Then, with the aim to evaluate the limits and the potential of this reaction we extended this reactivity to different acyl chlorides: this investigation led to the disclosure of a general and simple methodology to access selenolesters. ${ }^{25}$ Examples of this reactivity are summarized in Table 1.

As can be seen from the Table 1, reactions run smoothly with both aromatic (entries 1-4) and aliphatic acyl chlorides (entries 5 and 6). Interestingly, the reaction occurs efficiently even on functionalized acyl chlorides, such as $\alpha$-chloro propenoyl chloride (entry 6).

It is also noteworthy that the reactions can also be efficiently performed in the presence of phenoxide ion as the catalyst, with similar results.

Thus, having demonstrated the efficiency of selenosilanes in transferring a seleno moiety, we moved toward the functionalization of a more interesting molecule, hexa- 
methyldisilaselenane (HMDSS), ${ }^{26}$ that could have led to the synthesis of diacyl selenides, another related interesting class of compounds. Such molecules are very efficient transfer reagents for acyl and aroyl groups.

Table 1 Synthesis of Selenolesters 3 and ${ }^{77}$ Se NMR Chemical Shifts

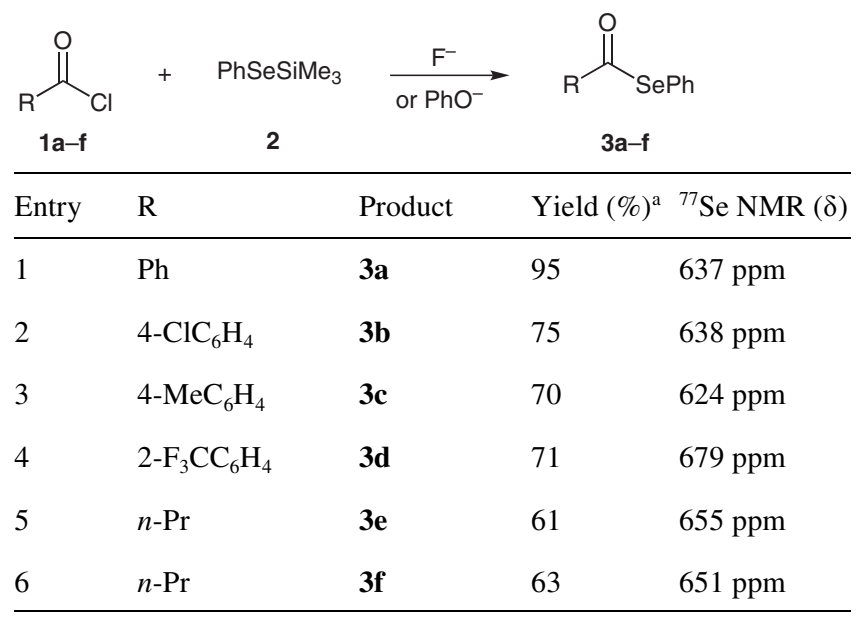

${ }^{\text {a }}$ Based on isolated compounds

Despite their synthetic interest, the synthesis of diacyl selenides and especially diacyl diselenides has been scarcely reported.

Some methods have been described along the years, ${ }^{27}$ but they are affected by several drawbacks, such as the use of expensive and unstable reagents, the limited availability of the starting materials, the difficulty in purification, particularly evident for diacyl selenides and diselenides, and the high number of steps required.

Diacyl selenides have been obtained from selenoamides, in turn obtained from arylnitriles and sodium hydroselenide, with acyl chlorides. The yields were satisfactory, but the method required a tedious preparation of selenoamides, which were very unstable. ${ }^{28}$ More recently Koketsu, Ishihara and co-workers reported the synthesis of LiAlHSeH a useful selenating reagent for the synthesis of a wide range of selenium-containing molecules that allowed an access in high yields and in a single step to diacyl selenides. ${ }^{29}$ Nonetheless LiAlHSeH is unstable and requires to be generated in situ and immediately used, thus somewhat limiting the procedure. Further oxidation of the so obtained selenoanhydrides with iodine afforded diacyl diselenides.

Thus, following the results obtained in the functionalization of phenylselenotrimethylsilane, we reacted two equivalents of benzoyl chloride (1a) with HMDSS (4) under the influence of TBAF (Scheme 2), to obtain a 75\% yield of dibenzoyl selenide (5a). So, HMDSS proved to be another interesting reagent for transferring a selenated moiety.

The reaction can be easily extended to different acyl chlorides, as reported in Table 2 (entries 1-5). ${ }^{30}$

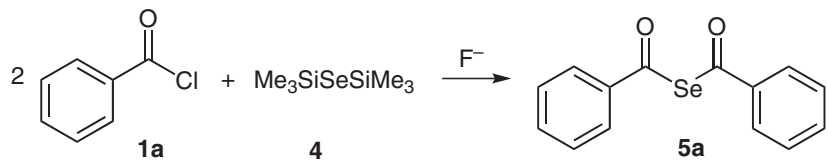

Scheme 2

Table 2 Preparation of Selenoanhydrides 5 and Diacyl Diselenides 6

\begin{tabular}{|c|c|c|c|c|}
\hline Entry & $\mathrm{R}$ & $\mathrm{RCOCl} / \mathrm{HMDSS}$ & Product & Yield (\%) \\
\hline 1 & $\mathrm{Ph}$ & $2: 1$ & $5 \mathbf{a}$ & 80 \\
\hline 2 & $4-\mathrm{ClC}_{6} \mathrm{H}_{4}$ & $2: 1$ & $5 \mathbf{b}$ & 76 \\
\hline 3 & $4-\mathrm{MeOC}_{6} \mathrm{H}_{4}$ & $2: 1$ & $5 c$ & 75 \\
\hline 4 & $\mathrm{MeCHCl}$ & $2: 1$ & $5 d$ & 50 \\
\hline 5 & 2-thienyl & $2: 1$ & $5 e$ & 70 \\
\hline 6 & $\mathrm{Ph}$ & $1: 1$ & $6 a$ & 83 \\
\hline 7 & $4-\mathrm{ClC}_{6} \mathrm{H}_{4}$ & $1: 1$ & $6 \mathbf{b}$ & 78 \\
\hline 8 & $4-\mathrm{MeOC}_{6} \mathrm{H}_{4}$ & $1: 1$ & $6 c$ & 71 \\
\hline 9 & $\mathrm{MeCHCl}$ & $1: 1$ & 6d & 54 \\
\hline 10 & 2-thienyl & $1: 1$ & $6 e$ & 73 \\
\hline
\end{tabular}

Even more interesting appeared the reactivity with stoichiometric ratios of acyl chlorides and HMDSS, that afforded in very good yields the corresponding diacyl diselenides (Scheme 3), ${ }^{31}$ probably arising from oxidation of the intermediate selenocarboxylic acid (derived from trimethylsilyl selenolester) in the workup procedure. ${ }^{32}$ Again, this methodology was extended to different substrates, thus showing its generality. Results are reported in Table 2 (entries 6-10).

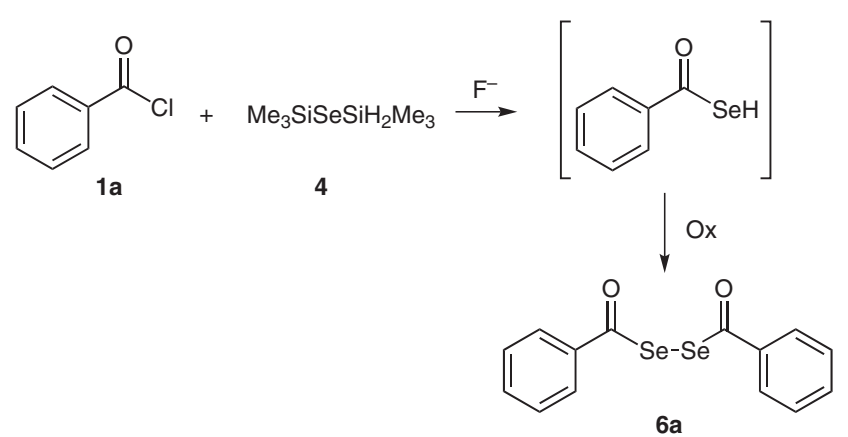

Scheme 3

Interestingly, no appreciable contamination was ever found between diacyl selenides and -diselenides in the aromatic series, thus affording a clean and selective entry to both classes of compounds that are known to be extremely difficult to separate.

In the case of the alkyl derivative, the diacyl diselenide $\mathbf{6 d}$ was contaminated by a small amount $(<10 \%)$ of the selenoanhydride $\mathbf{5 d}$. 
The ratio of $\mathbf{5 d}$ and $\mathbf{6 d}$ cannot be unequivocally obtained either from ${ }^{1} \mathrm{H}$ NMR or from ${ }^{13} \mathrm{C} \mathrm{NMR}$ spectra (the signals being very similar) but could nonetheless be easily obtained through their ${ }^{77} \mathrm{Se}$ NMR spectra, that show very typical ranges of absorption for the two classes of compounds 5 and $6 .{ }^{77} \mathrm{Se}$ NMR chemical shifts are collected in Table 3 .

Table 3 Typical ${ }^{77}$ Se NMR Chemical Shifts of Compounds 5 and 6

\begin{tabular}{lllll}
\hline $\mathrm{R}$ & \multicolumn{2}{l}{ Compound ${ }^{77} \mathrm{Se} \mathrm{NMR}$} & \multicolumn{2}{l}{ Compound ${ }^{77} \mathrm{Se} \mathrm{NMR}$} \\
& $\mathbf{5}$ & $(\delta)$ & $\mathbf{6}$ & $(\delta)$ \\
\hline $\mathrm{Ph}$ & $\mathbf{5 a}$ & $743 \mathrm{ppm}$ & $\mathbf{6 a}$ & $613 \mathrm{ppm}$ \\
$4-\mathrm{ClC}_{6} \mathrm{H}_{4}$ & $\mathbf{5 b}$ & $749 \mathrm{ppm}$ & $\mathbf{6 b}$ & $619 \mathrm{ppm}$ \\
$4-\mathrm{MeOC}_{6} \mathrm{H}_{4}$ & $\mathbf{5 c}$ & $730 \mathrm{ppm}$ & $\mathbf{6 c}$ & $598 \mathrm{ppm}$ \\
$\mathrm{MeCHCl}$ & $\mathbf{5 d}$ & $784 \mathrm{ppm}$ & $\mathbf{6 d}$ & $601 \mathrm{ppm}$ \\
2-thienyl & $\mathbf{5 e}$ & $777 \mathrm{ppm}$ & $\mathbf{6 e}$ & $614 \mathrm{ppm}$ \\
\hline
\end{tabular}

We also checked the reactivity toward diacyl chlorides, by reacting phthaloyl chloride (7) with HMDSS (Scheme 4) and we were able to isolate in $83 \%$ yield the corresponding selenoanhydride $\mathbf{8}$, thus showing that a smooth entry also to cyclic selenoanhydrides could be devised.

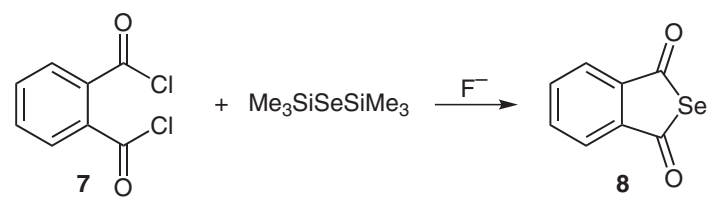

Scheme 4

It must anyway be stated that in these experiments, even when operating under different conditions, no traces of the benzo $[d][1,2]$ diselenine-1,4-dione could ever be detected; this probably means that the ring-closure reaction is faster than the selenation of the second acyl moiety.

Finally, with the aim to evaluate the efficiency of such a procedure also in different reaction media, acyl chlorides were reacted with HMDSS in ionic liquids. These compounds are in fact regarded as alternative and eco-friendly novel solvents of increasing interest. ${ }^{33}$

Thus, upon treatment of benzoyl chloride (1a) and 4-chlorobenzoyl chloride (1b) with 0.5 equivalent of HMDSS and $\mathrm{PhON}(n-\mathrm{Bu})_{4}(0.04$ equiv $)$ in $[\mathrm{bmim}]\left[\mathrm{PF}_{6}\right]$ at room temperature, a clean entry to the corresponding selenoanhydrides $\mathbf{5 a}$ and $\mathbf{5 b}$ was obtained with comparable yields with respect to the reaction in THF (Scheme 5).

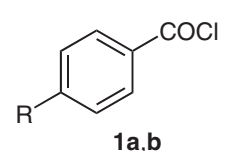

$1 a, b$
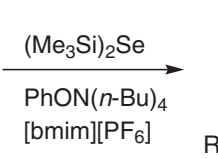

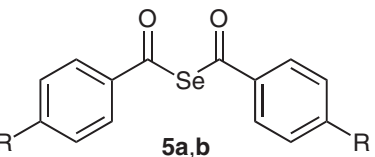

Scheme 5
To the best our knowledge, this represents the first example of syntheses of selenoanhydrides in ionic liquids, providing evidence of the versatility of this synthetic approach.

In conclusion, we have demonstrated that silylselenides behave as efficient transfer agents of selenated moieties, providing selectivity and good yields in rather mild conditions to selenolesters, diacyl selenides and diacyl diselenides.

\section{Acknowledgment}

Financial support from MiUR, National Project PRIN 2007 'Stereoselezione in Sintesi Organica. Metodologie ed Applicazioni' is gratefully acknowledged. Mrs. B. Innocenti is acknowledged for carrying out GC-MS analyses.

\section{References and Notes}

(1) For example, see: (a) Procter, D. J. J. Chem. Soc., Perkin Trans. 1 2000, 835. (b) Organoselenium Chemistry: A Practical Approach; Back, T. G., Ed.; Oxford University Press: Oxford, 1999. (c) Comasseto, J. V.; Ling, L. W.; Petragnani, N.; Stefani, H. A. Synthesis 1997, 373.

(d) Krief, A. Comprehensive Organometallic Chemistry, Vol. 11; Abel, E. W.; Stone, E. G. A.; Wilkinson, G., Eds.; Pergamon: Oxford, 1995, 515. (e) Krief, A.; Hevesi, L. Organoselenium Chemistry I; Springer: Berlin, 1988.

(2) See also: (a) Braga, A. L.; Lüdtke, D. S.; Vargas, F.; Braga, R. C. Synlett 2006, 1453. (b) Braga, A. L.; Vargas, F.; Sehnem, J. A.; Braga, R. C. J. Org. Chem. 2005, 70, 9021. (c) Braga, A. L.; Lüdtke, D. S.; Sehnem, J. A.; Alberto, E. E. Tetrahedron 2005, 61, 11664. (d) Braga, A. L.; Paixão, M. W.; Lüdtke, D. S.; Silveira, C. C.; Rodrigues, O. E. D. Org. Lett. 2003, 5, 3635.

(3) (a) Nogueira, C. W.; Zeni, G.; Rocha, J. B. T. Chem. Rev. 2004, 104, 6255. (b) Mugesh, G.; duMont, W.-W.; Sies, H. Chem. Rev. 2001, 101, 2125. (c) Nicolaou, K. C.; Petasis, N. A. Selenium in Natural Products Synthesis; CIS: Philadelphia, 1984; and references therein. (d) Klayman, D. L.; Günter, W. H. H. In Organoselenium Compounds: Their Chemistry and Biology; Wiley Interscience: New York, 1973.

(4) Pheninger, J.; Heuberger, C.; Graf, W. Helv. Chim. Acta 1980, 63, 2328.

(5) (a) Keck, G.; Grier, M. C. Synlett 1999, 1657. (b) Boger, D. L.; Mathvink, R. J. J. Org. Chem. 1992, 57, 1429. (c) Chen, C.; Crich, D.; Papadatos, A. J. Am. Chem. Soc. 1992, 114, 8313.

(6) Hiiro, T.; Morita, Y.; Inoue, T.; Kambe, N.; Ogawa, A.; Ryu, I.; Sonoda, N. J. Am. Chem. Soc. 1990, 112, 455.

(7) (a) Sviridov, A. F.; Ermolenko, M. S.; Yashunsky, D. V.; Kochetkov, N. K. Tetrahedron Lett. 1983, 24, 4355. (b) Anderson, R. J.; Henrick, C. A.; Rosenblum, L. D. J. Am. Chem. Soc. 1974, 96, 3654. (c) Mukaiyama, T.; Araki, M.; Takei, H. J. Am. Chem. Soc. 1973, 95, 4763.

(8) (a) McGarvey, G. J.; Williams, J. M.; Hiner, R. N.; Matsubara, Y.; Oh, T. J. Am. Chem. Soc. 1986, 108, 4943. (b) Conrow, R.; Portoghese, P. J. Org. Chem. 1986, 51, 938. (c) Back, T. G.; Kerr, R. G. Tetrahedron 1985, 41, 4759.

(9) (a) Suh, K.-H.; Choo, D.-J. Tetrahedron Lett. 1995, 36, 6109. (b) Kobayashi, S.; Uchiro, H.; Fujishita, Y.; Shiina, I.; Mukaiyama, T. J. Am. Chem. Soc. 1991, 113, 4247.

(10) Renson, M.; Draguet, C. Bull. Soc. Chim. Belg. 1962, 71, 260. 
(11) (a) Ren, K.; Wang, M.; Liu, P.; Wang, L. Synthesis 2010, 1078. (b) Movassagh, B.; Mirshojaei, F. Monatsh. Chem. 2003, 134, 831. (c) Chen, R.; Zhang, Y. Synth. Commun. 2000, 30, 1331. (d) Zang, Y.; Yu, Y.; Lin, R. Synth. Commun. 1993, 23, 189.

(12) (a) Zhang, S. L.; Tian, F. S.; Li, G. A.; Wang, Y. L. Chin. Chem. Lett. 2002, 13, 9. (b) Xu, X. H.; Liu, W. Q. Chin. Chem. Lett. 2002, 13, 283. (c) Wang, L.; Zhang, Y. Synth. Commun. 1999, 29, 3107.

(13) (a) Wallner, O. A.; Szabo, K. J. J. Org. Chem. 2005, 70, 9215. (b) Nishiyama, Y.; Kawamatsu, H.; Funato, S.; Tokunaga, K.; Sonoda, N. J. Org. Chem. 2003, 68, 3599. (c) Nishiyama, Y.; Tokunaga, K.; Kawamatsu, H.; Sonoda, N. Tetrahedron Lett. 2002, 43, 1507. (d) Beletskaya, I.; Sigeev, A. S.; Peregudov, A. S.; Petrovskii, P. V. Russ. J. Org. Chem. 2001, 37, 1703. (e) Schiesser, C. H.; Skidmore, M. A. J. Chem. Soc., Perkin Trans. 1 1997, 2689.

(14) (a) Tiecco, M.; Testaferri, L.; Temperini, A.; Bagnoli, L.; Marini, F.; Santi, C.; Terlizzi, R. Eur. J. Org. Chem. 2004, 3447. (b) Braga, A. L.; Martins, T. L. C.; Silveira, C. C.; Rodrigues, O. E. Tetrahedron 2001, 47, 3297. (c) Hoshi, M.; Masuda, Y.; Arase, A. Bull. Chem. Soc. Jpn. 1988, 61, 3764.

(15) Grieco, P. A.; Yokoyama, Y.; Williams, E. J. Org. Chem. 1978, 43, 1283.

(16) (a) Marin, G.; Braga, A. L.; Rosa, A. L.; Galetta, F. Z.; Burrow, R. A.; Gallardo, H.; Paixão, M. W. Tetrahedron 2009, 65, 4614. (b) Munbunjong, W.; Lee, E. H.; Ngernmaneerat, P.; Kim, S. J.; Singh, G.; Chavasiri, W.; Jang, D. O. Tetrahedron 2009, 65, 2467. (c) Braga, A. L.; Schneider, P. H.; Paixão, M. W.; Deobald, A. M. Tetrahedron Lett. 2006, 47, 7195. (d) Ranu, B. C.; Mandal, T. J. Org. Chem. 2004, 69, 5793.

(17) Tabarelli, G.; Alberto, E. E.; Deobald, A. M.; Marin, G.; Rodrigues, O. E. D.; Dornelles, L.; Braga, A. L. Tetrahedron Lett. 2010, 51, 5278.

(18) (a) Dan, W.; Deng, H.; Chen, J.; Liu, M.; Ding, J.; Wu, H. Tetrahedron 2010, 66, 7384. (b) Qian, H.; Shao, L.-X.; Huang, X. Synlett 2001, 1571. (c) Fujiwara, S.-I.; Asai, A.; Shinike, T.; Kambe, N.; Sonoda, N. J. Org. Chem. 1998, 63, 1724. (d) Inoue, T.; Takeda, T.; Kambe, N.; Ogawa, A.; Ryu, I.; Sonoda, N. J. Org. Chem. 1994, 59, 5824. (e) Kozikowski, A. P.; Ames, A. Tetrahedron 1985, 41, 4821. (f) Grieco, P. A.; Jaw, J. Y.; Claremon, D. A.; Nicolau, K. C. J. Org. Chem. 1981, 46, 1215. (g) Back, T. G.; Collins, S.; Kerr, R. G. J. Org. Chem. 1981, 46, 1564.

(19) (a) Degl'Innocenti, A.; Pollicino, S.; Capperucci, A. Chem. Commun. 2006, 4881. (b) Degl'Innocenti, A.; Capperucci, A.; Malesci, I.; Castagnoli, G.; Acciai, M.; Nocentini, T.; Pollicino, S. Synlett 2006, 2439. (c) Degl'Innocenti, A.; Capperucci, A. Eur. J. Org. Chem. 2000, 2171.

(d) Capperucci, A.; Degl'Innocenti, A.; Leriverend, A.; Metzner, P. J. Org. Chem. 1996, 61, 7174. (e) Capperucci, A.; Ferrara, M. C.; Degl'Innocenti, A.; Bonini, B. F.; Mazzanti, G.; Zani, P.; Ricci, A. Tetrahedron Lett. 1994, 35, 16. (f) Capperucci, A.; Ferrara, M. C.; Degl'Innocenti, A.; Bonini, B. F.; Mazzanti, G.; Zani, P.; Ricci, A. Synlett 1992, 880. (g) Ricci, A.; Degl'Innocenti, A.; Mordini, A.;

Reginato, G.; Colotta, V. Gazz. Chim. It. 1987, 117, 645. (h) Ricci, A.; Degl'Innocenti, A.; Chimichi, S.; Fiorenza, M.; Rossini, G.; Bestman, H. J. J. Org. Chem. 1985, 50, 130.

(20) (a) Capperucci, A.; Tiberi, C.; Pollicino, S.; Degl'Innocenti, A. Tetrahedron Lett. 2009, 50, 2808. (b) Capperucci, A.; Degl'Innocenti, A.; Pollicino, S.; Acciai, M.; Castagnoli, G.; Malesci, I. Heteroat. Chem. 2007, 18, 516.

(c) Degl'Innocenti, A.; Capperucci, A.; Nocentini, T. Tetrahedron Lett. 2000, 42, 4557.
(21) (a) Degl'Innocenti, A.; Capperucci, A.; Castagnoli, G.; Malesci, I. Synlett 2005, 1965. (b) Bouillon, J.-P.; Capperucci, A.; Portella, C.; Degl'Innocenti, A. Tetrahedron Lett. 2004, 45, 87. (c) Capperucci, A.; Degl'Innocenti, A.; Nocentini, T.; Biondi, S.; Dini, F. J. Organomet. Chem. 2003, 686, 363. (d) Capperucci, A.; Degl'Innocenti, A.; Biondi, S.; Nocentini, T.; Rinaudo, G. Tetrahedron Lett. 2003, 44, 2831. (e) Degl'Innocenti, A.; Capperucci, A.; Oniciu, D.; Katritzky, A. R. J. Org. Chem. 2000, 65, 9206. (f) Degl'Innocenti, A.; Capperucci, A.; Scafato, P.; Mecca, T.; Reginato, G.; Mordini, A. Synlett 1999, 1739. (g) Ricci, A.; Degl'Innocenti, A.; Capperucci, A.; Reginato, G. J. Org. Chem. 1989, 54, 19.

(22) Degl'Innocenti, A.; Capperucci, A.; Cerreti, A.; Pollicino, S.; Scapecchi, S.; Malesci, I.; Castagnoli, G. Synlett 2005, 3063.

(23) Degl'Innocenti, A.; Capperucci, A.; Acciai, M.; Tiberi, C. Phosphorus, Sulfur Silicon Relat. Elem. 2009, 184, 1621.

(24) Degl'Innocenti, A.; Capperucci, A.; Castagnoli, G.; Malesci, I.; Tiberi, C.; Innocenti, B. Phosphorus, Sulfur Silicon Relat. Elem. 2008, 183, 966.

(25) Preparation of Se-Phenyl 2-chloropropaneselenoate (3f): To a solution of 2-chloropropanoyl chloride $(30 \mathrm{mg}$, $0.24 \mathrm{mmol})$ in anhyd THF $(0.5 \mathrm{~mL})$ was added under an inert atmosphere phenylselenotrimethylsilane $(71 \mu \mathrm{L}, 0.28$ mmol). TBAF ( $50 \mu \mathrm{L}, 1 \mathrm{M}$ THF solution, $0.05 \mathrm{mmol})$ was added dropwise at $0{ }^{\circ} \mathrm{C}$. The reaction was warmed to r.t. and stirred overnight. The mixture was then extracted with $\mathrm{Et}_{2} \mathrm{O}$, washed with brine and dried over $\mathrm{Na}_{2} \mathrm{SO}_{4}$. Filtration and evaporation of the solvent gave the crude $\mathbf{3 f}$ as a yellow oil, which was purified by TLC on silica gel $\left(n\right.$-hexane- $\mathrm{CH}_{2} \mathrm{Cl}_{2}$, 2:1). Yield: $63 \% .{ }^{1} \mathrm{H}$ NMR (200 MHz, $\left.\mathrm{CDCl}_{3}\right): \delta=1.75$ (d, $3 \mathrm{H}, J=7.0 \mathrm{~Hz}), 4.58(\mathrm{q}, 1 \mathrm{H}, J=7.0 \mathrm{~Hz}), 7.43-7.46(\mathrm{~m}, 3$ $\mathrm{H}), 7.51-7.55(\mathrm{~m}, 2 \mathrm{H}) .{ }^{13} \mathrm{C}$ NMR $\left(50 \mathrm{MHz}, \mathrm{CDCl}_{3}\right): \delta=$ $22.1,63.7,127.5,129.3,130.6,131.3,200.4 .{ }^{77} \mathrm{Se}(38 \mathrm{MHz}$, $\left.\mathrm{CDCl}_{3}\right): \delta=650.6$. MS: $m / z(\%)=248(26)\left[\mathrm{M}^{+}\right], 192(22)$, 158 (39), 91 (44), 78 (55), 63 (100).

(26) (a) Lange, H.; Herzog, U. J. Organomet. Chem. 2002, 660, 36. (b) Detty, M. R.; Seidler, M. D. J. Org. Chem. 1982, 47, 1354.

(27) (a) Niyomura, O.; Tani, K.; Kato, S. Heteroat. Chem. 1999, 10, 373. (b) Kageyama, H.; Kido, K.; Kato, S.; Murai, T. J. Chem. Soc., Perkin Trans. 1 1994, 1083. (c) Kato, S.; Kageyama, H.; Kawahara, Y.; Murai, T.; Ishihara, H. Chem. Ber. 1992, 125, 417. (d) Wang, J.-X.; Wang, C.-H.; Wang, W.-F.; Cui, W.-F.; Hu, Y.-L.; Zang, S.-S. J. Chem. Res., Synop. 1990, 230. (e) Nishiyama, Y.; Katsuura, A.; Okamoto, Y.; Hamanaka, S. Chem. Lett. 1989, 18, 1825. (f) Ishihara, H.; Kato, S. Tetrahedron Lett. 1972, 3751. (g) Jensen, K. A.; Bøje, L.; Henriksen, L. Acta Chem. Scand. 1972, 26, 1465.

(28) (a) Zhao, H.-R.; Zhao, X.-J.; Huang, X. Synth. Commun. 2002, 32, 3383. (b) Zhao, H.-R.; Zhao, X.-J.; Huang, X. Chin. Chem. Lett. 2001, 12, 873.

(29) (a) Koketsu, M.; Nada, F.; Hiramatsu, S.; Ishihara, H. J. Chem. Soc., Perkin Trans. 1 2002, 737. (b) Ishihara, H.; Koketsu, M.; Fukuta, Y.; Nada, F. J. Am. Chem. Soc. 2001, 123,8408 .

(30) Synthesis of 4-Methoxybenzoic Selenoanhydride (5c): A solution of 4-methoxybenzoyl chloride $(24 \mu \mathrm{L}, 0.18 \mathrm{mmol})$ and TBAF ( $1 \mathrm{M}$ in THF, $18 \mu \mathrm{L}, 0.018 \mathrm{mmol}$ ) in anhyd THF $(0.5 \mathrm{~mL})$ was cooled under atmosphere at $0{ }^{\circ} \mathrm{C}$, and treated dropwise with bis(trimethylsilyl)selenide (HMDSS; $21 \mathrm{mg}$, $0.09 \mathrm{mmol})$. The red mixture turned to pale yellow after few minutes. After warming to r.t., and stirring for $2 \mathrm{~h}$, the solution was diluted with $\mathrm{Et}_{2} \mathrm{O}$, washed with brine and dried over $\mathrm{Na}_{2} \mathrm{SO}_{4}$. The crude product was purified by flash 
column chromatography ( $n$-hexane- $\mathrm{CH}_{2} \mathrm{Cl}_{2}, 1: 1$ ) to afford 5c as a yellow solid $(24 \mathrm{mg}, 75 \%) .{ }^{1} \mathrm{H}$ NMR $(200 \mathrm{MHz}$, $\left.\mathrm{CDCl}_{3}\right): \delta=3.88(\mathrm{~s}, 6 \mathrm{H}), 6.93-6.98(\mathrm{~m}, 4 \mathrm{H}), 7.92-7.96(\mathrm{~m}$, $4 \mathrm{H}) .{ }^{13} \mathrm{C} \mathrm{NMR}\left(50 \mathrm{MHz}, \mathrm{CDCl}_{3}\right): \delta=55.6,114.1,130.9$, $131.5,164.5,185.9 .{ }^{77} \mathrm{Se}\left(38 \mathrm{MHz}, \mathrm{CDCl}_{3}\right): \delta=730.0$.

(31) Preparation of 4-Methoxybenzoic Diselenoperoxyanhydride (6c): A solution of 4-methoxybenzoyl chloride $(24 \mu \mathrm{L}, 0.18 \mathrm{mmol})$ in anhyd THF $(0.5 \mathrm{~mL})$ was added under an inert atmosphere with HMDSS (44 mg, $0.19 \mathrm{mmol}$ ) and it was cooled to $0{ }^{\circ} \mathrm{C}$. TBAF $(1 \mathrm{M}$ in THF, $38 \mu \mathrm{L}, 0.038$ $\mathrm{mmol}$ ) was then added dropwise. The red mixture so formed was warmed to r.t. and stirred for $2 \mathrm{~h}$. Upon quenching with brine, the red solution turned slowly to pale yellow.

Extraction with $\mathrm{Et}_{2} \mathrm{O}$, drying over $\mathrm{Na}_{2} \mathrm{SO}_{4}$ and removal of the solvent under vacuum afforded the crude product, which was purified by flash column chromatography ( $n$-hexane-
$\left.\mathrm{CH}_{2} \mathrm{Cl}_{2}, 1: 1\right)$ to produce $\mathbf{6 c}$ as a yellow solid (55 $\left.\mathrm{mg}, 71 \%\right)$. ${ }^{1} \mathrm{H}$ NMR $\left(200 \mathrm{MHz}, \mathrm{CDCl}_{3}\right)$ :

$\delta=3.88(\mathrm{~s}, 6 \mathrm{H}), 6.92-6.98(\mathrm{~m}, 4 \mathrm{H}), 7.91-8.07(\mathrm{~m}, 4 \mathrm{H}) \cdot{ }^{13} \mathrm{C}$ $\operatorname{NMR}\left(50 \mathrm{MHz}, \mathrm{CDCl}_{3}\right): \delta=55.6,114.2,129.3,130.5$,

164.4, 186.9. ${ }^{77} \mathrm{Se}\left(38 \mathrm{MHz}, \mathrm{CDCl}_{3}\right): \delta=598.5$.

(32) Kageyama, H.; Murai, T.; Kanda, T.; Kato, S. J. Am. Chem. Soc. 1994, 116, 2195.

(33) See also: (a) Sawant, A. D.; Raut, D. G.; Darvatkar, N. B.; Salunkhe, M. M. Green Chem. Lett. Rev. 2011, 4, 41. (b) Greaves, T. L.; Drummond, C. J. Chem. Rev. 2008, 108, 206. (c) Chowdhury, S.; Mohan, R. S.; Scott, J. L. Tetrahedron 2007, 63, 2363. (d) Sheldon, R. Chem. Commun. 2001, 2399. (e) Wasserscheid, P.; Keim, W. Angew. Chem. Int. Ed. 2000, 39, 3772. (f) Welton, T. Chem. Rev. 1999, 99, 207. 